\title{
DISEÑO DE UNA INTERVENCIÓN FORMATIVA CON MAPAS CONCEPTUALES EN COMUNIDADES DE APRENDIZAJE
}

\section{DESIGN OF A FORMATIVE INTERVENTION USING CONCEPT MAPS IN LEARNING COMMUNITIES}

\author{
Dra. Pilar Ibáñez-Cubillas \\ pcubillas@ugr.es \\ Dra. Da María-Jesús Gallego-Arrufat \\ mgallego@ugr.es
}

\begin{abstract}
Departamento de Didáctica y Organización Escolar. Facultad de Ciencias de la Educación. Universidad de Granada. Campus de Cartuja, s/n. 18071 Granada, España.
\end{abstract}

\begin{abstract}
Resumen: El estudio describe una intervención formativa con mapas conceptuales en Educación Superior. Se dirige a analizar las potencialidades y mejorar las prácticas que permitan dinamizar los procesos formativos en comunidades de aprendizaje, mediante la aplicación de dos cuestionarios y un protocolo ad hoc para analizar los mapas. Para ello se analizan las necesidades formativas percibidas por los participantes para usar los mapas conceptuales como herramienta de aprendizaje, se lleva a cabo una intervención formativa, se comparan los mapas elaborados por los estudiantes en comunidades con los de profesorado experto y se determina el nivel de satisfacción al finalizar la intervención. Se sigue una metodología que responde a una Investigación Basada en el Diseño (IBD) en la que se experimenta con 75 estudiantes universitarios de Pedagogía una intervención sistemática centrada en el empleo de las Tecnologías de la Información y Comunicación (TIC) para la elaboración de mapas conceptuales. Como resultado, se detecta la necesidad de formación para una elaboración correcta de los mapas, así como una diferencia cualitativa importante entre los mapas elaborados por los estudiantes y el mapa modelo experto. También aparece un alto grado de satisfacción entre los participantes en el uso de mapas conceptuales. Palabras clave: Mapas conceptuales, satisfacción, necesidades formativas, comunidades de aprendizaje.
\end{abstract}

Abstract: The study describes a formative intervention using concept maps in Higher Education. It aims to analyze potentialities and improve practices to dynamize formative processes in learning communities through administration of two questionnaires and an ad hoc protocol to analyze the maps. To fulfill these goals, we analyze the formative needs perceived by the participants in order to use concept maps as a learning tool. We then perform a formative intervention, compare the maps developed by the students in communities to those of the expert professors, and determine the satisfaction level once the intervention is complete. We follow a design-based research method (DBR) to experiment with 75 university students in Education, performing a systematic intervention that focuses on the use of Information and Communication Technologies (ICT) to develop concept maps. As a result, we find the need for training to develop the maps correctly, as well as a significant qualitative difference between the students' maps and the expert model. We also find a high degree of satisfaction among the participants regarding the use of concept maps.

Keywords: Concept mapping, satisfaction, training needs, communities of practice.

$$
-97-
$$

Píxel-Bit. Revista de Medios y Educación. No 51 Julio 2017. ISSN: 1133-8482. e-ISSN: 2171-7966. doi: http://dx.doi.org/10.12795/pixelbit.2017.i51.06 


\section{Introducción.}

Los mapas conceptuales son una herramienta versátil empleada en numerosos contextos (Freeman \& Jessup, 2004) para representar el conocimiento de las personas y así hacer explícito el conocimiento tácito (Chiou, Lee \& Liu, 2012; Lee \& Seveg, 2012). En educación, los mapas conceptuales son empleados para la enseñanza y el aprendizaje de los estudiantes universitarios. A comienzos de los noventa, el estudio de Heinze-Fry \& Novak (1990) encontró un aumento del logro. Posteriormente, aparecen efectos positivos derivados del uso de los mapas conceptuales en numerosas investigaciones (Adesope \& Nesbit, 2009; De Benito, Darder \& Salinas, 2012; Gallego, Crisol \& Gámiz, 2013; Kinchin, 2014).

Aunque proliferan herramientas y servicios para elaborar mapas conceptuales, reconocemos la fundamentación de Novak (2013) quien promueve los mapas conceptuales basados en la teoría del aprendizaje significativo de Ausubel, en la que un nuevo aprendizaje es relacionado con el conocimiento ya existente, permitiendo que quede incluido en la estructura cognitiva, y dando lugar así a nuevo aprendizaje. Por lo tanto, los conceptos ya existentes permiten un «anclaje» en los nuevos conceptos para ser incorporados a la estructura cognitiva, facilitando el aprendizaje significativo. Los mapas conceptuales son representaciones esquemáticas sobre significados conceptuales incluidos en una estructura de proposiciones (Novak \& Cañas, 2008). Son representaciones en diagrama de un conjunto de conceptos-clave relacionados entre sí. Los conceptos se representan en una estructura jerárquica y las líneas que se trazan entre los conceptos formando proposiciones muestran visualmente estas relaciones (Hinck et al, 2006; Valdés, Menéndez \& Valdés Pardo, 2006).

En este estudio optamos por utilizar un modelo jerarquizado (Novak \& Cañas, 2008; Novak, 2013). Se establece de este modo una estructura restrictiva que distingue a los mapas conceptuales de otras herramientas de representación del conocimiento (con estructura proposicional, jerarquía, pregunta de enfoque y enlaces cruzados). Los mapas son una herramienta versátil, empleada y extendida para representar el conocimiento de los sujetos y hacerlo explícito -incluso en el caso del conocimiento experto, de carácter tácito- así como para promover el aprendizaje a través de la propia creación y reflexión (Valdés, Menéndez \& Valdés Pardo, 2006; Gallego, Crisol \& Gámiz, 2013).

Cada vez es más fácil, mediante diferentes programas, aplicaciones y herramientas, la elaboración de los mapas conceptuales. En su origen Knowledge Manager o Inspiration, y en la actualidad aplicaciones como Xmind, SmartDraw, FreeMind, Text2 MindMap, junto a otras aplicaciones multiplataforma con apps para el smartphone o iPad como SimpleMind+, Popplet, o combinadas con servicios de uso compartido como Google Drive, Dropbox y OneDrive (Creately, Coogle, GoConqr). En este estudio utilizamos el software CmapTools <http:// cmap.ihmc.us/>, por ser el más documentado en la investigación educativa para mejorar la comprensión y habilidad para aprender a aprender significativamente, tanto de forma individual como colaborativa (Novak \& Cañas, 2008; Adesope \& Nesbit, 2009; Kinchin, 2014). Se trata de una herramienta que permite construir, navegar y compartir modelos de conocimiento mediante mapas conceptuales, los cuales pueden ser

$$
-98-
$$


elaborados por varias personas, colaborando de forma síncrona o asíncrona, al mismo tiempo que ven el proceso y los cambios conforme avanzan (Novak, 2013; Kinchin, 2014).

Esta funcionalidad colaborativa que ofrece el software, permite que los estudiantes trabajen cooperativamente en pequeños grupos para elaborar su mapa conceptual mediante CmapTools y, hacen uso de ellos para guiar este proceso, lo que produce un aprendizaje significativo mayor. Al trabajar los contenidos de forma colaborativa y online, se crean comunidades de práctica en entornos virtuales, definidas como "grupo de personas que comparten una preocupación, un conjunto de problemas o un interés común acerca de un tema, y que profundizan su conocimiento y pericia en esta área a través de una interacción continuada» (Wenger, McDermott \& Snyder, 2002, p.4). Comunidades donde la interacción y cooperación entre los miembros generan resultados cognitivos y afectivos positivos que resultan de la comunicación y diálogo social como apoyo al aprendizaje (Preszler, 2004).

Junto a esto, a pesar de los múltiples beneficios que proporcionan las comunidades de práctica en contextos virtuales y la elaboración de mapas conceptuales de forma colaborativa, la satisfacción de los estudiantes es más relevante si se desea su mantenimiento en el tiempo. Aunque existen investigaciones en las que los estudiantes ponen de relieve la satisfacción ante el uso de los mapas conceptuales empleados en determinadas situaciones, los estudiantes de Educación usuarios de mapas conceptuales muestran una alta satisfacción en su empleo, no sólo como herramienta de aprendizaje, sino también de evaluación (Buldu \& Buldu, 2010). De hecho, Hinck et al. (2006) obtienen evidencias de la satisfacción de los estudiantes a través de un cuestionario cuyos resultados indican un aprendizaje significativo y un grado de satisfacción elevado.

Sin embargo, algunos estudios sobre mapas conceptuales evidencian las dificultades de uso generalizado de esta herramienta en relación con la satisfacción mostrada por los estudiantes. También se encuentra que algunos estudiantes se sienten más cómodos, más satisfechos y obtienen mejor rendimiento que otros en el uso de mapas conceptuales o con algunos elementos de la actividad (GonzálezBrignardello, Sánchez-Elvira, \& AmorAndrés, 2010). Hinck et al. (2006) afirman que el grado de satisfacción en el aprendizaje de los estudiantes puede estar condicionado por los diferentes estilos de aprendizaje.

En este contexto, los estudios centrados en las necesidades formativas permiten generar y orientar los programas de formación, aunque «en la enseñanza universitaria es poco frecuente realizar una evaluación sobre necesidades formativas» en cualquier materia (Rodríguez Gallego, 2002, p.109). Existe una gran variedad de modelos para la detección de necesidades formativas aplicables en distintos contextos formativos. En todo caso, la detección de necesidades debe respetar el contexto donde se realiza y hacia quién se dirige, asumiendo las necesidades como carencias percibidas que pueden ser cubiertas mediante procesos formativos (Rodríguez Gallego, 2002). El conocimiento exhaustivo de las necesidades orienta y dirige la propuesta formativa basada en las exigencias y en el entorno. Se ha puesto de manifiesto que la mejora del aprendizaje

-99 -

Píxel-Bit. Revista de Medios y Educación. $N^{\circ} 51$ Julio 2017. ISSN: 1133-8482. e-ISSN: 2171-7966. doi: http://dx.doi.org/10.12795/pixelbit.2017.i51.06 
es más difícil en personas que no son conscientes de sus deficiencias.

En general, las investigaciones se centran en los efectos del uso y elaboración de mapas en el aprendizaje de los estudiantes, pero no muestran los detalles de su formación. Una excepción es el estudio de Hinck et al. (2006), con estudiantes de Enfermería, que incluye un curso para promover el desarrollo de conocimientos y habilidades organizándose un taller previo orientado a satisfacer las expectativas de los estudiantes y sus necesidades, y aprender en la práctica la creación de mapas conceptuales. Las necesidades identificadas se basan en las dificultades de los estudiantes en la creación de los mapas conceptuales y en la representación a través del software, así como en su uso.

\section{Método.}

\subsection{Problema y objetivos del estudio}

El problema surge de la necesidad de dinamizar los procesos de enseñanza y aprendizaje en educación superior, mediante la identificación de las potencialidades y prácticas con tecnologías, como es el caso de la elaboración de mapas conceptuales digitales en comunidades de aprendizaje. Se distinguen los siguientes objetivos:

1. Detectar las necesidades formativas, reales y percibidas, que presentan los estudiantes sobre el uso y elaboración de mapas conceptuales en formato digital.

2. Experimentar intervenciones formativas para validar la integración de prácticas basadas en el uso y elaboración de mapas conceptuales digitales.

3. Determinar el grado de satisfacción, expectativas y opiniones de los estudiantes sobre el uso y elaboración de mapas conceptuales digitales en el proceso de enseñanza y aprendizaje.

Reconocemos la importancia y la pertinencia de integrar los mapas conceptuales y las tecnologías emergentes para su elaboración en entornos de aprendizaje, que permitan obtener implicaciones formativas para la mejora de su utilización.

\subsection{Diseño de la investigación}

En este estudio la metodología responde a una investigación basada en el diseño (Design-Based Research Collective, 2003; Van den Akker et al., 2006) centrada en un contexto universitario de aprendizaje de tecnologías de la información y comunicación en Pedagogía. Se diseña y experimenta una intervención sistemática centrada en el empleo de tecnologías en comunidades de aprendizaje. En este caso, se analiza la relación entre el aprendizaje significativo sobre el que descansa el empleo de los mapas conceptuales digitales junto a dos variables: la necesidad de formación y la satisfacción percibidas. Centrados en el diseño y desarrollo de la intervención, en su estructura y resultados, así como en el modo de análisis de proceso y resultado derivado del empleo de los mapas, adopta la forma de una investigación basada en el diseño (IBD) que responde a un tipo de investigaciones caracterizadas como rigurosas y reflexivas para probar y perfeccionar los entornos de aprendizaje innovadores, así como para definir nuevos principios de diseño (Amiel \& Reeves, 2008). La validez de la investigación se considera según las dimensiones de efectividad, eficiencia y significado (Reigeluth \& Frick, 1999).

$$
-100-
$$


Los participantes son un total de 75 estudiantes del grado de Pedagogía (63 mujeres y 12 hombres), matriculados en la asignatura de «Tecnología Educativa» de la Universidad de Granada, en la que se trabajan contenidos relacionados con el aprendizaje, aplicación e integración de las TIC en los procesos educativos.

El diseño del proceso de intervención se dirige a potenciar la eficacia de las comunidades online en tres ámbitos, en una estrategia formativa adaptada del modelo $3 \mathrm{C}$ de colaboración. Las 3Cs son: Comunicación (facilita la puesta en común de conocimientos), Comunidad (ayuda a integrar comunidades) y Cooperación (permite generar conocimiento compartido) (Fuks, Raposo \& Gerosa, 2008). El uso de recursos dentro y fuera del entorno Moodle (foros, enlaces web, bancos de imágenes y vídeo, redes sociales), de acceso abierto y licencias Creative commons, fomentan las 3 Cs. Durante las primeras sesiones de la asignatura, los estudiantes de pedagogía se organizaron por grupos de 5 a 6 miembros y se distribuyeron entre los tres seminarios de prácticas. También al comienzo se administró el Cuestionario sobre el uso de mapas conceptuales. Detección de necesidades formativas, a través de la plataforma institucional Prado2 (Moodle). Posteriormente, en el ecuador de la asignatura, se habilitó en la plataforma un espacio para la interacción entre los estudiantes donde tratar las cuestiones concernientes al diseño de los mapas electrónicos. A continuación, recibieron una formación en elaboración de mapas conceptuales y el uso de CmapTools, previa a la realización de un mapa conceptual colaborativo por parte de los estudiantes, sobre una temática concreta y mediante el uso de este software. Una vez concluido el periodo de formación y el tiempo dedicado al desarrollo de la actividad, un representante de cada grupo compartió su mapa conceptual con el resto de la clase a través del espacio generado en la plataforma, convertido en una comunidad de prácticas. Tras las entrega de la actividad se pidió al alumnado que completara el Cuestionario sobre satisfacción en el uso de mapas conceptuales, con el fin de medir el grado de satisfacción tras la intervención didáctica. Una vez concluido, se procedió al análisis de la información recogida de los cuestionarios y de la parrilla de análisis, para continuar con la extracción de las conclusiones.

En este proceso, la intervención educativaincluye seis pasos: creación de las comunidades en grupo empleando los foros de la asignatura en Moodle; aplicación del cuestionario de necesidades formativas; formación en mapas conceptuales; realización del mapa en comunidades; aplicación delcuestionario de satisfacción y; análisis de los datos y conclusiones. Una vez que los estudiantes se agruparon en comunidades a través de los foros generados en Moodle, se aplicó el cuestionario de necesidades formativas entre los estudiantes y se continuó con el diseño de intervención para la formación previa en la elaboración de los mapas conceptuales y el uso del software CmapTools. Antes de comenzar con la intervención educativa, se trabajaron en clase los contenidos relativos a las «metodologías activas para la formación con tecnologías» correspondientes al manual básico (GallegoArrufat \& Raposo-Rivas, 2016), haciendo uso de los mapas conceptuales digitales como estrategia de enseñanza. Una vez concluido dicho tema, se informó a los estudiantes que deberían elaborar un mapa conceptual sobre

$-101-$

Píxel-Bit. Revista de Medios y Educación. No 51 Julio 2017. ISSN: 1133-8482. e-ISSN: 2171-7966. doi: http://dx.doi.org/10.12795/pixelbit.2017.i51.06 
los contenidos trabajados, actividad que se realizaría en los grupos creados al comienzo de la asignatura.A continuación, se dedicaron los tres seminarios siguientes a la formación en mapas conceptuales y al uso de CmapTools, aportando documentación adicional. En el campusinstitucional de la universidad, Prado2 (Moodle), se crearon comunidades de prácticas a través de foros, donde los miembros de los grupos intercambiaban información. También se podía apreciar la evolución y desarrollo que habían seguido algunos de los mapas conceptuales, hasta compartir el definitivo con el resto de la clase a través de este espacio. Para el diseño del mapa conceptual digital, los grupos llevaron a cabo una serie de tareas previas que eran tan importantes como el producto final debido a los altos niveles de rendimiento cognitivo que se ponen en juego (Novak \& Cañas, 2008): a) lectura del material, b) selección de los conceptos clave, c) puesta en común y selección de los conceptos clave identificados en el paso anterior, c) se consensúa la relevancia de los conceptos, colocando el más general en la parte superior del mapa conceptual y los conceptos más inclusivos debajo, d) se comienza a realizar el mapa conceptual preliminar buscando la relación entre los conceptos, que serán unidos a través de conectores y palabras de enlace, e) estudian, debaten y modifican el mapa conceptual hasta que es consensuado por todos, f) diseño final del mapa conceptual. Una vez que los mapas conceptuales electrónicos fueron entregados por los grupos, se volvió activar el Cuestionario sobre satisfacción en el uso de mapas conceptuales, con el fin de conocer el grado de satisfacción tras la formación y elaboración del mapa conceptual. Con la cumplimentación del cuestionario se daba por concluida la intervención educativa para proceder al análisis de los datos, las conclusiones y las sugerencias de mejora.

\subsection{Instrumentos de recogida y análisis de información}

Se emplean dos cuestionarios como instrumentos de recogida de datos cuantitativos, junto a un protocolo de análisis de contenido para los datos cualitativos.

El instrumento «Cuestionario sobre el uso de mapas conceptuales. Detección de necesidades formativas» se estructura en dos partes: la primera con variables de identificación y, la segunda parte con diez ítems (con opciones de respuesta Sí-No), divididos en tres dimensiones: formación, estructura académica y financiación.E1 «Cuestionario sobre satisfacción en el uso de mapas conceptuales» tiene tres secciones: la primera es información estadística, y la segunda parte está compuesta por tres dimensiones: Conocimiento y uso anterior (sobre mapas conceptuales y CmapTools); Uso futuro (el futuro uso que harán de CmapTools y los mapas conceptuales según su satisfacción); y Formación (formación en mapas conceptuales recibida y el software utilizado). La tercera parte corresponde al grado de satisfacción y son frases positivas (con escala Likert, de valores 1: Nada a 5: Totalmente) que aluden a las dimensiones mencionadas.

El contenido de ambos cuestionarios fue anteriormente validado en función de la univocidad (exactitud), pertinencia e importancia (1-3) por cinco jueces expertos. Se optó por calcular el ICC (obteniendo un aceptable .584 y .483 respectivamente) y se realizaron los cambios y/o sugerencias

$$
-102-
$$


señaladas por los revisores. Se obtuvo su fiabilidad en un estudio piloto realizado en una actividad formativa ofertada a todos los estudiantes matriculados en la Facultad de Ciencias de la Educación de la UGR. La fiabilidad de los cuestionarios fue determinada por el Alpha de Cronbach; $(\mathrm{a}=.719 \mathrm{y} \mathrm{a}=.837$ respectivamente).

Para el análisis cualitativo de los mapas electrónicos elaborados por los estudiantes se diseñó una parrilla con un sistema categorial ad hoc, compuesto por nueve dimensiones agrupadas en tres categorías: a) estructura del mapa conceptual; b) errores estructurales y c) aplicación de los recursos CmapTools. La categoría estructura del mapa incluye tres aspectos: (1) Revisión de las líneas de conexión entre los conceptos. Analiza el uso correcto de flechas conectoras como elementos direccionales; (2) Número de conceptos establecidos por el estudiante. Se valora el número de conceptos incluidos por los estudiantes para poder determinar, a partir del mapa del experto, si el estudiante incluyó todos los conceptos relevantes, si añadió más conceptos a partir de su conocimiento previo o por el contrario algunos no son relevantes; (3) Número de niveles de los conceptos o jerarquización. Por la importancia de la jerarquización, desde esta dimensión se valora la posición que toman los conceptos de acuerdo al contenido. La categoría errores estructurales atiende a tres aspectos: (1) Repetición de conceptos: es erróneo repetir conceptos, pues éstos sólo deben aparecer una vez en el mapa conceptual. (2) Confusión entre conceptos y conectores: la confusión o una errónea selección de éstos no permitiría un aprendizaje significativo. (3) Lectura comprensiva y coherente: el mapa conceptual debe de ser leído con coherencia pudiendo reconstruir un texto con sentido completo.
La categoría aplicación de los recursos CmapToolscomprende tres elementos según el uso y dominio del software: (1) Información adicional incluida, en cualquier modalidad (audio, vídeo, texto...). (2) Inclusión de imágenes, ya sea de fondo o como información aportada a los conceptos. (3) Colores y estilos de letra, para enfatizar los conceptos relevantes y hacer más atractivo el mapa conceptual.

Una vez aplicados los instrumentos y realizado el vaciado de datos, se realizó un análisis estadístico descriptivo de los cuestionarios (basado en medidas de centralización y dispersión) y un análisis del contenido de los mapas según la parrilla de análisis descrita.

\section{Resultados.}

Los resultados obtenidos responden a las necesidades formativas, el resultado de la aplicación del protocolo de análisis de mapas digitales, y la satisfacción de los participantes. Atendiendo a los resultados obtenidos en los cuestionarios iniciales, un porcentaje muy elevado conocía los mapas conceptuales y los había utilizado alguna vez, sin embargo, se obtuvieron evidencias de sus carencias formativas debidas al mal uso de conectores, la ausencia de palabras de enlace o la propia confusión de los conceptos, hallada con la aplicación de la parrilla de análisis.

\subsection{Necesidades formativas de los estudiantes sobre el uso de mapas conceptuales digitales}

El resultado de la categoría «formación» muestra la necesidad percibida por los estudiantes de formación en mapas. El 72.5\%

- 103 -

Píxel-Bit. Revista de Medios y Educación. $N^{\circ} 51$ Julio 2017. ISSN: 1133-8482. e-ISSN: 2171-7966. doi: http://dx.doi.org/10.12795/pixelbit.2017.i51.06 
considera que los profesionales de la educación no cuentan con este tipo de

formación, y que deberían tener una formación amplia en mapas conceptuales (92.2\%), así como en el uso del software CmapTools (94.1\%). Sobre la formación en mapas conceptuales digitales dentro de los estudios reglados y su oferta formativa dentro de la propia institución universitaria, reflejan una opinión dividida, según el resultado de la categoría «estructura académica» (Figura 1).

Para el 51\% la oferta formativa en mapas conceptuales es suficiente y para el $49 \%$ no lo es, y mientras que el $51 \%$ de estudiantes indica que esta formación debe ser impartida en las titulaciones universitarias oficiales, el $49 \%$ considera preferible mantenerla al margen de las titulaciones.Por otro lado, el $62.7 \%$ de los participantes considera que la formación en mapas conceptuales debería ofrecerse dentro de cada asignatura, frente al $35.3 \%$ que indica lo contrario. Más concretamente, para un $84.3 \%$ la formación debería de impartirse en el primer semestre del curso inicial pero el $15.7 \%$ no. Un $94.1 \%$ piensa que la formación en mapas conceptuales debería ser complementaria y con posibilidad de reconocimiento de créditos y, solo el 5.9\% considera lo contrario.

La categoría «conocimiento y uso anterior» muestra hasta qué punto los mapas conceptuales digitales son conocidos por los estudiantes, prestando atención a herramientas informáticas para su elaboración. Antes de ser presentados en la asignatura, $90.2 \%$ señalan que los conocen y $74.5 \%$ que los han utilizado con anterioridad. Aproximadamente la mitad afirman conocerlos (52.9\% frente a $47.1 \%$ no) y han hecho uso del programa CmapTools en algún momento anterior ( $51 \%$ frente a $49 \%$ no). El $80.4 \%$ no conoce otro programa para la elaboración de mapas conceptuales, frente al $17.6 \%$ que menciona otro tipo de aplicaciones (ej. Prezzi).

\subsection{Comparación con mapa de experto mediante parrilla de análisis}

La información de carácter más cualitativo según la parrilla de análisis para los mapas conceptuales y su sistema de categorías,

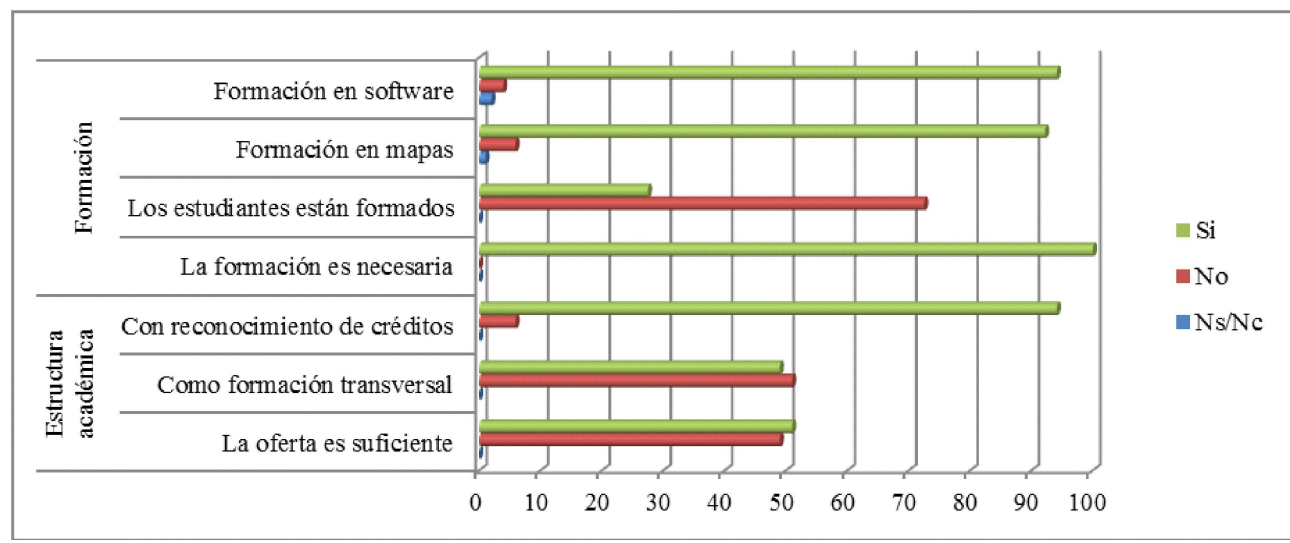

Figura 1. Detección de necesidades formativas: Categorías «formación» y «estructura académica»

Fuente: Elaboración propia

$-104-$

Píxel-Bit. Revista de Medios y Educación. No 51 Julio 2017. ISSN: 1133-8482. e-ISSN: 2171-7966. doi: http://dx.doi.org/10.12795/pixelbit.2017.i51.06 
evidencia la elaboración intuitiva de los participantes en contraste con un mapa de experto. El mapa del experto cuenta con la revisión de flechas (es decir, el concepto se une a la palabra de enlace mediante línea y éste se une al siguiente concepto mediante flecha). Está compuesto por 17 conceptos organizados en tres niveles. El experto no incluye información adicional sobre el texto proporcionado, no hace uso de imágenes para ilustrar la información, ni emplea otro tipo de letras o colores diferentes a los establecidos inicialmente por el programa.

Comparando los mapas de los grupos y del experto, se detecta que la utilización de las flechas y líneas es realizada debidamente en 9 mapas, mientras que el resto (6) no muestran un uso correcto en su totalidad. Existe menor número de conceptos en los mapas de los estudiantes (entre 17 y 7 , media $=12$ conceptos), observándose gran diversidad en el número de conceptos reconocidos (desviación típica $=5.17$ ). En cuanto al número de niveles, los estudiantes están próximos al mapa experto, estableciendo 3 ó 4 niveles (media $=4$ y desviación típica $=1.6)$. Tan sólo en dos casos repiten conceptos y en 4 de los 15 mapas se detecta confusión entre conceptos y conectores.

En cuanto a la lectura de los mapas digitales, es comprensiva y coherente en la mayoría de los casos (11 mapas). Mientras que el experto no utiliza imágenes ni colores y no incluye información adicional, los estudiantes noveles lo hacen con mucha frecuencia, incluyendo 13 mapas información adicional. Todos emplean imágenes y usan colores y estilos de letra distintos a los predeterminados por el programa.

\subsection{Grado de satisfacción de los estudiantes universitarios sobre el uso de mapas conceptuales digitales}

La categoría «futuro uso» aporta como resultado que el $78.4 \%$ de los estudiantes usarán los mapas conceptuales como herramienta de estudio, 98\% recomendarían el uso de mapas conceptuales y $96.1 \%$ utilizarán el programa. Además, según la categoría «formación», la mayor parte de los estudiantes $(84.3 \%)$ consideran necesaria una base teórica, frente al $15.7 \%$ que cree que no.

En cuanto a los resultados sobre satisfacción en el uso de mapas conceptuales, los estudiantes indican que hacen bastante uso de ellos ( $\mathrm{x}=3.40$, desviación típica 0.81 ), correspondiéndose al mismo nivel con el grado de mejora y utilidad para el aprendizaje percibido ( $\mathrm{x}=3.67$, desviación típica 0.68 ). Señalan que es muy necesaria la formación en mapas conceptuales $(x=3.59$, desviación típica 0.75$)$, y que su uso presenta muy poca dificultad ( $x=2.75$, desviación típica 0.90$)$.

\section{Discusión.}

La satisfacción de los estudiantes en el uso de los mapas conceptuales digitales es básica, porque en educación con tecnologías la satisfacción de los usuarios ante un instrumento, producto o proceso es el principal criterio para su uso continuado (Freeman \& Jessup, 2004). La mejora y la innovación en la enseñanza no sólo dependen del profesorado sino también en gran medida de la participación y el compromiso de los estudiantes, y para ello es previo contar con un nivel de satisfacción adecuado.

Los resultados obtenidos muestran que los estudiantes presentan una elevada

- 105 -

Píxel-Bit. Revista de Medios y Educación. No 51 Julio 2017. ISSN: 1133-8482. e-ISSN: 2171-7966. doi: http://dx.doi.org/10.12795/pixelbit.2017.i51.06 
satisfacción en el uso y elaboración de los mapas conceptuales como herramienta para el estudio, así como en el uso del software CmapTools. Se debe tener en cuenta que, con anterioridad, los mapas conceptuales eran conocidos por $90.2 \%$ y usados por $74.5 \%$ de participantes, lo cual indica que la satisfacción no se relaciona con la novedad de la herramienta, sino con los beneficios que les aporta en su estudio. Por otro lado, la herramienta CmapTools tan sólo es conocida y utilizada por el $51 \%$ de los estudiantes. También aparece un porcentaje elevado de desconocimiento de otras herramientas para elaborar mapas conceptuales. Los estudiantes consideran necesaria una formación para el conocimiento de la herramienta de estudio y del software. Es también elevado el dato sobre la posible recomendación de los mapas conceptuales (78.4\%), junto a la recomendación de uso del software CmapTools (96.1\%), aunque el desconocimiento de otras herramientas probablemente limite el alcance de este resultado. De modo similar, Hinck et al. (2006) mostraron que los estudiantes aportaban comentarios positivos sobre los mapas conceptuales como tarea, al ser diferentes, interesantes y permitir recordar otros conceptos, y además como herramienta de aprendizaje en sí misma. Es más, Freeman \& Jessup (2004), indican que los mapas conceptuales son una gran herramienta de comunicación, útil y fácil de usar, mientras que Preszler (2004) afirma que los procesos asociados con la construcción de mapas conceptuales basados en el aprendizaje cooperativo mejora la capacidad de los estudiantes para acceder a la información y utilizarla para responder a preguntas desafiantes.
Desde la propia percepción de los estudiantes, como en el estudio de Didi ${ }^{\circ}$, Özcan \& Azar (2014), se contempla la necesidad de una formación sobre el empleo y construcción de mapas conceptuales electrónicos. Consideran que esta formación no se encuentra entre los profesionales de la educación y que por tanto sería necesaria. Chiou, Lee \& Liu(2012) señalan la importancia y la dificultad que comprende formar en la realización de mapas conceptuales, ya que la percepción de los estudiantes en su estudio incluye la dificultad de adaptarse rápidamente a los mapas conceptuales en un periodo corto de tiempo. También en el presente estudio se ha confirmado la necesidad de formación y capacitación en el uso de esta herramienta. Los resultados obtenidos suponen una respuesta efectiva de los estudiantes ya que implica la necesidad de contar con una formación eficaz en el uso de mapas conceptuales digitales para apoyar el proceso de enseñanza y aprendizaje.

Examinados los resultados de la parrilla de análisis de mapas elaborados por estudiantes y su comparación con el análisis del mapa realizado por un experto, se aprecia una diferencia considerable entre ambos que apunta a necesidades reales de formación. Esto permite identificar que los participantes elaboran mapas conceptuales de forma intuitiva y sugerir que una formación previa proporcionaría una mayor efectividad en el proceso de aprendizaje. Por tanto, la percepción de los estudiantes ante la necesidad de una formación sobre mapas conceptuales digitales coincide con la detección de carencias formativas en la elaboración de sus mapas conceptuales.

Como conclusión, tras un riguroso análisis con los instrumentos descritos, esta investigación basada en el diseño de la

$$
\text { - } 106 \text { - }
$$


intervención formativa pone de manifiesto que para los participantes los mapas conceptuales son una herramienta de estudio con la que se encuentran satisfechos. Del mismo modo, atendiendo a los resultados, es posible sugerir que una formación continuada sobre el uso y elaboración de los mapas conceptuales mejoraría la capacidad de aprendizaje de los estudiantes y les situaría en un nivel de elaboración más próximo al del experto.

Estudios como el presente se consideran una aportación útil para crear y mejorar teorías de diseño instructivo (Reigeluth \& Frick, 1999), a través de intervenciones educativas innovadoras mejoradas, que descansan en la secuencia necesidad formativa-formaciónexperiencia educativa-satisfacciónsugerencias de mejora interactivas. El trabajo en comunidades de aprendizaje orientadas a la indagación con tecnologías (mapas conceptuales u otras, con CMapTools u otras herramientas) necesita futuras investigaciones. La investigación en Tecnología Educativa necesita generar un tipo de conocimiento válido que pueda ser útil para profesorado, estudiantes, educadores y miembros de la comunidad educativa, lo cual nos sitúa en una «investigación formativa», es decir, un tipo de investigación de desarrollo o investigación de acción que tiene como objetivo mejorar la teoría del diseño para diseñar prácticas o procesos formativos (Reigeluth \& Frick, 1999). De ahí que estimamos que las investigaciones similares a este tipo de estudios de diseño, en la que colaboran investigadores y profesionales, profesores y estudiantes, puede mejorar el diseño, implementación y análisis de diferentes clases de situaciones, incluyendo el uso de tecnologías como recursos. Se sugiere continuar realizando estudios empíricos en esta línea, cercana a la investigación formativa, para profundizar en la mejora pedagógica, didáctica y metodológica del empleo de mapas conceptuales en Educación Superior que apoyen la estructuración y organización de las ideas clave para la construcción de sitios web, presentaciones multimedia o la preparación y ejecución de un proyecto en Web 2.0, competencias digitales básicas en el espacio europeo.

\section{Fuentes de financiación}

Programa de Formación de Profesorado Universitario (F.P.U.) Referencia: FPU13/ 04744

\section{Referencias bibliográficas}

Adesope, O. O., \& Nesbit, J. C. (2009). A systematic review of research on collaborative learning with concept maps. In Lupion Torres, P. (Ed.), Handbook of research on collaborative learning using concept mapping (pp. 238-255).USA: IGI Global.

Amiel, T., \& Reeves, T. C. (2008). DesignBased research and educational technology: Rethinking technology and the research agenda. Educational Technology \& Society, 11(4). Recuperado de https://goo.gl/EXuwY

Buldu, M.,\& Buldu, N. (2010). Concept mapping as a formative assessment in college classrooms: Measuring usefulness and student satisfaction. Procedia- Social and Behavioral Sciences, 2(2), 2009-2104. doi:10.1016/j.sbspro.2010.03.288

Chiou, C. C., Lee, L. T., \& Liu, Y. Q. (2012). Effect of Novak colorful concept map with digital teaching materials on student academic achievement. Procedia-Social and

- $107-$

Píxel-Bit. Revista de Medios y Educación. $N^{\circ} 51$ Julio 2017. ISSN: 1133-8482. e-ISSN: 2171-7966. doi: http://dx.doi.org/10.12795/pixelbit.2017.i51.06 
Behavioral Sciences, 64, 192-201. doi:10.1016/ j.sbspro.2012.11.023

De Benito, B., Darder, A., \& Salinas, J. (2012). Los itinerarios de aprendizaje mediante mapas conceptuales como recurso para la representación del conocimiento. Edutec, Revista Electrónica de Tecnología Educativa, 39. Recuperado de https://goo.gl/ kyufDv

Design-Based Research Collective. (2003). Design-based research: An emerging paradigm for educational inquiry. Educational Researcher, 32 (1). Recuperado de http://www.jstor.org/stable/3699927

Didi ${ }^{\circ}$, N., Özcan, Ö., \& Azar, A. (2014). What do pre-service physics teachers know and think about concept mapping? Eurasia Journal of Mathematics, Science \& Technology Education, 10 (2), 77-87. doi:10.12973/eurasia.2014.1031a

Freeman, L. A., \& Jessup, L. M. (2004). The power and benefits of concept mapping: measuring use, usefulness, ease of use, and satisfaction. International Journal of Science Education, 26 (2), 151-169. doi:10.1080/ 0950069032000097361

Fuks, H., Raposo, A., \& Gerosa, M. A. (2008). The $3 \mathrm{c}$ collaboration model.In Encyclopedia of E-collaboration (pp. 637644). USA: IGI Global.

Gallego, M.J., Crisol,E.,\& Gámiz, V.(2013).El mapa conceptual como estrategia de aprendizaje y de evaluación en la universidad. $\mathrm{Su}$ influencia en el rendimiento de los estudiantes. Enseñanza \& Teaching, 31(2). Recuperado de https://goo.gl/8n7S9D

Gallego-Arrufat, M. J., \& Raposo-Rivas, M. (2016). Formación para la educación con tecnologías. Madrid: Pirámide.

González-Brignardello, M.P., SánchezElvira, A. \& Amor-Andrés, P.J. (2010). Elaboración de mapas conceptuales: relación con estilos de aprendizaje, rendimiento y satisfacción. En Concept Maps: Making Learning Meaningful. Fourth Int. Conference on Concept Mapping (pp.90-94). Viña del Mar, Chile.

Heinze-Fry, J. A., \& Novak, J. D. (1990). Concept mapping brings long-term movement toward meaningful learning. Science Education, 74(4), 461-472. doi:10.1002/ sce. 3730740406

Hinck, S.M., Webb, P., Simns-Giddens, S., Helton, C., Hope, K., Utley, O., Savinske, D., Fahey, E. \& Yarbrough, S. (2006). Student learning with concept mapping of care plans in community-based education. Journal of Professional Nursing, 22(1), 23-29. doi:10.1016/j.profnurs.2005.12.004

Kinchin, I. M. (2014). Concept mapping as a learning tool in higher education: A critical analysis of recent reviews. The Journal of Continuing Higher Education, 62(1), 3949.doi:10.1080/07377363.2014.872011

Lee, J.H.,\& Segev, A. (2012).Knowledge maps for e-learning.Computers \& Education, 59(2), 353-364. doi:10.1016/ j.compedu.2012.01.017

Novak, J.D. (2013). Empowering learners and educators. Journal for Educators, Teachers and Trainers, 4(1). Recuperado de https://goo.gl/ezEpyY

Novak, J. D., \& Cañas, A. J. (2008). The theory underlying concept maps and how to construct and use them. Florida Institute for Human and Machine Cognition. Recuperado de https://goo.gl/x0mrNU

Preszler, R. (2004). Cooperative Concept Mapping: Improving Performance in Undergraduate Biology. Journal of College Science Teaching, 33(6), 30-35. Recuperado de https://goo.gl/DmjNeJ

Reigeluth, C. \& Frick, T. (1999). Formative research: A methodology for creating and

$$
-108-
$$


improving design theories. En C. Reigeluth, (Ed.), Instructional-Design theories and models. A new paradigm of instructional theory (pp. 633-652). Mahwah (NJ), USA: Lawrence Erlbaum.

Rodríguez Gallego, M. (2002). Necesidades formativas de los alumnos andaluces de magisterio en nuevas tecnologías de la información y la comunicación. Pixel Bit. Revista de Medios y Educación, 19. Recuperado de https://goo.gl/iM8hto

Valdés, M.A., Menéndez, L.M., \& Valdés Pardo, V.G. (2006). Los mapas conceptuales: un recurso para el aprendizaje apoyado en tecnologías. Pixel Bit. Revista de Medios y Educación, 28. Recuperado de https:// goo.gl/0sCFAl

Van den Akker, J., Gravemeijer, K., McKenney, S., \& Nieveen, N. (Eds.) (2006). Educational design research. London: Routledge.

Wenger, E., McDermott, R., \& Snyder, W. M. (2002). Seven principles for cultivating communities of practice. Cultivating communities of practice: A guide to managing knowledge, 4. Recuperado de https://goo.gl/tKIjBt

Fecha de recepción: 10/03/2017

Fecha de evaluación: 16/03/2017

Fecha de aceptación: 28/03/2017

- 109 -

Píxel-Bit. Revista de Medios y Educación. No 51 Julio 2017. ISSN: 1133-8482. e-ISSN: 2171-7966. doi: http://dx.doi.org/10.12795/pixelbit.2017.i51.06 